\title{
Life-Support Model of Supply Systems of Oil and Gas Industry Objects
}

\author{
Fares Abu-Abed ${ }^{1, *}$, and Alexey Khabarov ${ }^{1}$ \\ ${ }^{1}$ Tver State Technical University, A. Nikitin Street, 22, 170026, Tver, Russia
}

\begin{abstract}
At present, repair and diagnostic complexes are being developed for repair of oil and gas production facilities, including control areas for working capacity, diagnosing components used in maintenance stations, as well as areas equipped with the necessary technological equipment, measuring instruments, tools and equipment for carrying out current repair of replacement elements. For maintenance of repair, repair-diagnostic complexes are equipped with means of power supply, sets of spare property and accessories and operational documentation. Repair-diagnostic complexes can be represented using a three-phase multi-channel queuing system. The study of such a system with the distribution functions of the service time of applications different from the exponential makes it difficult to use analytical models. The developed simulation model of the repair and diagnostic complex allows to evaluate the characteristics of its functioning, the most important of which is the average service time of the application in the system, as well as to determine the required number of channels on each site. The developed model can be aggregated into a general model of research into the operation of complex technical systems for supplying oilfield facilities.
\end{abstract}

\section{Introdction}

The process of elimination of failures and malfunctions of complex technical systems includes a preliminary check of operability, identification of a failure (refuse) - control, localization of the fault location - diagnostics and elimination of failure - repair. Replacement elements (RE), i.e. the applications in case of their actual inoperability are consistently monitored by serviceability, diagnostics for the purpose of localizing the malfunction and directly repairing with the appropriate equipment and a system of spare assets and accessories [1]. Repaired REs are repeatedly tested for serviceability, and if a fault is detected, the process is repeated. If the repair is successful, the solar cells are returned to replenish the expended sets of maintenance spare parts.

Thus, the repair and diagnostic complex (RDC) can be represented as a multiphase, multichannel queuing system (Figure 1):

\footnotetext{
Corresponding author: $\underline{\text { aafares@mail.ru }}$
} 
The RE coming from the oil and gas industry are an input stream for the RDC. Workable replacement elements that fall on the input stream of the RDC for a false fault registration probability $\left(P_{\text {false }}\right)$ immediately fall on the output stream. The rest consistently pass the control, diagnostics and repair on the relevant sites.

Each of the sections consists of several lines. Repaired replacement elements with probability $\left(P_{\text {repair }}\right)$ at the input after repeated monitoring go to the output flow, and with probability to the diagnostic site and, subsequently, to the repair site, etc.

Multiphase mass service systems consist of several typical nodes arranged in series, i.e. are a collection of several queuing systems.

All requests served in one node are forwarded to the next node. In other words, the output stream of one node of a multiphase queuing system is the input stream for the next [2].

An exact calculation of the characteristics of such queuing systems is possible only if all streams of orders are Poisson, and all service times are random variables distributed according to an exponential law. In other cases, only an approximate calculation of the characteristics of the queuing system is possible.

When calculating the characteristics of multiphase queuing systems, the following [3] should be taken into account:

- if several streams of requests enter the input of the queuing system, then the intensity of the full flow of applications in this queuing system is equal to the sum of the intensities of the individual flows;

- if the input of the queuing system receives a part of the requests from a certain stream, whose intensity is equal to $\lambda$, then the intensity of the input stream of requests in the queuing system can be determined by the formula:

$\lambda_{\text {input }}(t)=P \lambda(t)$, where $P$ is the probability of the bid input stream;

- the intensity of the output stream in the queuing system (that is, the flow of served orders) is equal to the intensity of the input stream.

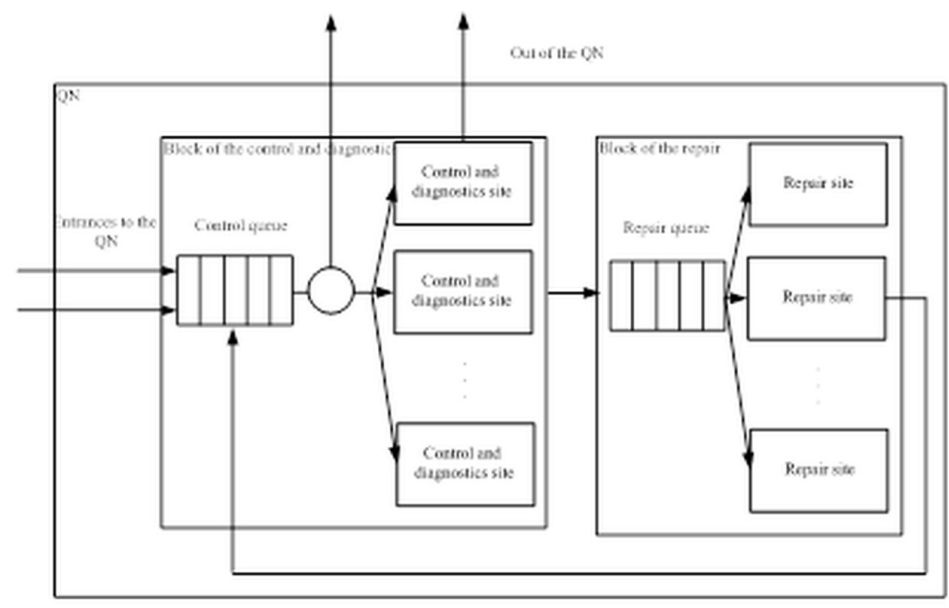

Fig. 1. Presentation of the RDC of a three-phase queuing system.

Applications received for servicing in the queuing system form a stream of applications. Elements of the queuing system, serving orders, create service channels.

In most cases, the time intervals between the points of receipt of applications and/or the times of service of requests in queuing systems are random variables, i.e. in most cases it is not known exactly in advance when the next application will be received and how long it will take for it to be serviced. Therefore, the theory of queuing systems is based on the mathematical apparatus of probability theory and mathematical statistics. 
The most accurate calculation of the characteristics is possible for queuing systems, in which the flow of applications is Poisson (simplest). The Poisson flow of applications has the properties of recurrence, stationarity, ordinary and no aftereffect.

If, under these conditions, the time for servicing applications at each site is subject to an exponential law, then in this case the queuing system model is easily described by a system of differential equations [4].

However, in practice, the service time can be more adequately represented using the Beta distribution.

\section{Materials and Methods}

Rationale for selecting the distribution law of duration of work performance in models of mass service systems

In queuing systems in which the maintenance process is subject to random influences, it is assumed that the duration of the work is a random variable.

It is assumed that the random values of the duration of work are subject to the distribution law accepted for the given queuing system, and the type of distribution is assumed to be the same for all works [5].

As for the distribution parameters, the latter are assigned for each work by their responsible executors on the basis of either normative data, or a priori considerations, or their own production experience.

In some queuing systems, three parameters are set: the lower bound $a$ of the definition area (optimistic time), the upper face $b$ (pessimistic time), and the mode of distribution $m$ (the most probable time).

Practically in all queuing systems it is a priori assumed that the distribution density of time estimates of the duration of work has three properties: a) continuity, b) unimodality, c) two nonnegative points of intersection of this density with the abscissa axis. The simplest distribution with similar properties is the Beta distribution, which is usually postulated in practice. The general view of the beta distribution is characterized, in addition to the presence of a large number of random factors, each of which separately has a slight, insignificant effect, the presence of several, also random, factors, the number of which is small, and the effect is significant.

As a result of the impact of significant factors, the probability distribution is usually made asymmetric. It is this kind of circumstance that takes place when the vast majority of the works included in the network project are realized [6].

This implies the possibility of choosing the Beta distribution as an a priori model.

The analysis of a large number of statistical data (timing of the implementation of individual works, regulatory data, etc.) also confirms the possibility of using the beta distribution as a priori.

General view of the beta distribution is characterized, in addition to having a large number of random factors, each of which individually have little, insignificant influence, the presence of several, also random factors, the number of which is small, but the effect is material. As a result of the influence of significant factors probability distribution is usually asymmetric. Just such a situation occurs in the implementation of the vast majority of members of the network project work. This implies the possibility to choose a beta distribution as a priori model. The analysis of large amounts of statistical data (duration time of the individual works implementation, normative data, and so on) also confirms the possibility of using the beta distribution as a priori. Formula of beta-distribution density is as follows:

$$
B(p, q, x)=\left\{\begin{array}{l}
\frac{1}{B(p, q)} x^{p-1} \text { if } 0 \leq \mathrm{x} \leq 1 \\
0 \text { if } x<0, x>1
\end{array}\right.
$$


where $B(p, q)$ - beta-function, with

$$
B(p, q)=\int_{0}^{1} x^{p-1}(1-x)^{q-1} d x=\frac{G(p) G(q)}{G(p+q)}
$$

and the gamma-function $\mathrm{G}(\mathrm{z})$ is defined by the formula $G(z)=\int_{0}^{\infty} e^{-t} t^{z-1} d t$,

and for integer values z gamma-function $G(z)=1 \times 2 \times \ldots \times(z-1)=(z-1)$ ! Initial moment of the $\mathrm{r}$-th order is determined by the formula:

$$
\frac{1}{B(p, q)} \int_{0}^{1} x^{r+p-1}(1-x)^{q-1} d x=\frac{B(p+r, q)}{B(p, q)}
$$

when $r=1$ the mathematical expectation

$$
M x=\frac{p}{p+q}
$$

when $r=2$ the dispersion

$$
D x=\frac{p q}{(p+q)^{2}(p+q+1)}
$$

The probability density of operation time, distributed according to the law of the beta distribution on the interval $[\mathrm{a}, \mathrm{b}]$ is $[4]$ :

$$
f(t)= \begin{cases}\frac{1}{(b-a)^{\alpha+\beta-1} B(\alpha, \beta)}(t-a)^{\alpha-1}(b-t)^{\beta-1} & \text { if } a \leq t \leq b \\ 0, & \text { otherwise. }\end{cases}
$$

For example, for the construction of probability theory apparatus of PERT, considered that the duration of any work is a random variable distributed according to the law of the beta distribution on the interval $[\mathrm{a}, \mathrm{b}]$, and the parameters of the distribution law - mean $\mathrm{M}$ and variance $\sigma^{2}$ - defined by the formulas:

$$
M=\frac{a+4 m+b}{6}, \quad \sigma^{2}=\frac{(b-a)^{2}}{36}
$$

where $a, b$ and $m$ - respectively, optimistic, pessimistic, and most likely (mode) evaluation, establishes by responsible executives of work.

Life cycle of the system. After the entry of the application into the queuing system, it is possible with a predetermined probability to terminate the service cycle and exit from the RDC. Upon the exit from the repair subsystem, applications are returned to the control subsystem, with a fixed probability of continuing service or leaving the RDC.

The number of places in the drives of each node is unlimited, all subsystems without failures. In the simulated queuing system, the discipline of the queue is used - the principle according to which the incoming requests to the service system are selected from the service queue, defined by the FIFO rule (First Input, First Output) [7].

In the existing implementation of the simulation model, the model time is progressed discretely, according to the principle of advancing the model time to the next event.

Model time is shifted forward not by a fixed amount, but precisely until the time of the earliest of the next events that has a number of advantages: events are considered and modeled at the time of their accomplishment, and simultaneously, if they have the same appearance time. Intervals of time, when nothing happens in the model, are skipped without special expenditure of computer time [8].

The possible variant shifts the time upon the occurrence of major - leading to changes in the system state - event has the form shown in figure 2 .

Timing diagrams for this Queuing Networks, representing the model time shift in accordance with the occurrence of events on this basis will be as follows: 

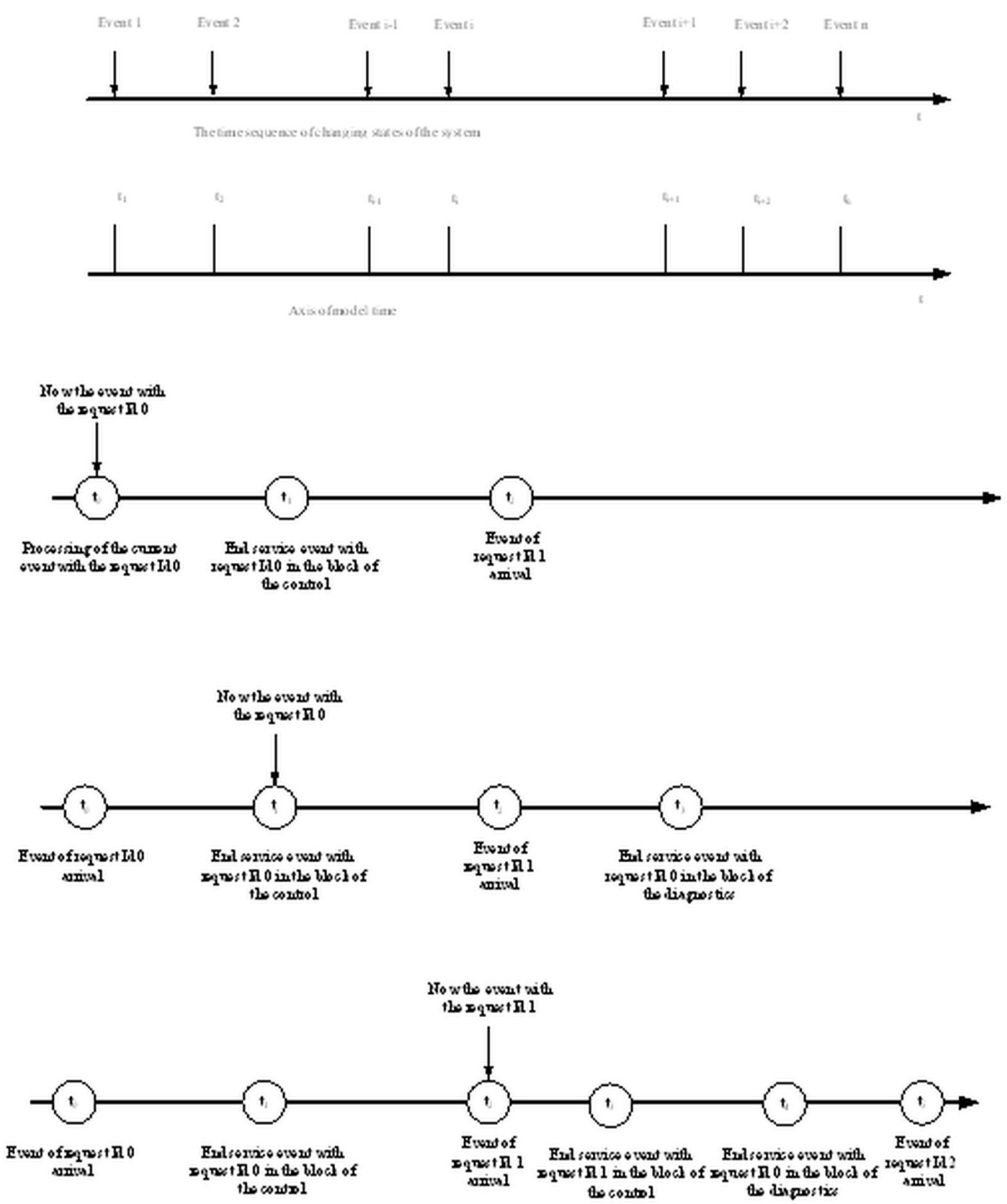

Fig. 2. Model time shift.

Algorithm of modeling of functioning of repair-diagnostic complex. When implementing a software model of a queuing system, it is advisable to use an object-oriented approach.

When each main event that is simulated by selecting the nearest event from the list of current events occurs, the model time shifts and the event is removed from the list as processed.

\section{Results and Discussion}

Description of work with the developed software RDC. Data input is carried out in the form of integers or fractional numbers, depending on the parameters that these data characterize. 
Integer and fractional numbers can be used to initialize the distribution coefficients, probabilities, and simulation time. When specifying the number of devices, we can only enter integer values (Figure 3).

Fractional numbers can be specified using a period or a comma between fractional and integer parts, however, if you enter more than one coefficient (which is necessary when initializing some distributions), you need to specify fractional numbers through a comma separated delimiter between the specified parameters.

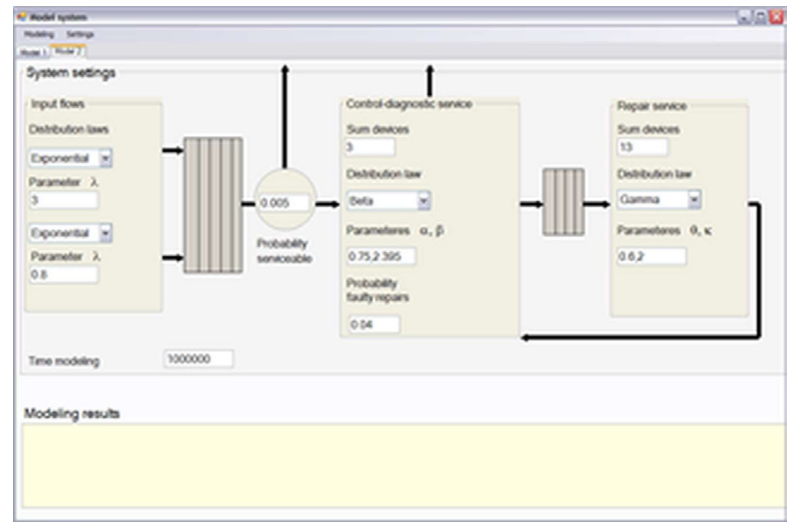

Fig. 3. The interface of the developed software.

The text box below the information input interface displays information about the current state of the application: a record of the need to wait for the simulation process to finish and, at its end, the results of the model operation.

Taking into account the principles of constructing models in the GPSS environment [1, 10], the algorithm for simulating the process of functioning of a given queuing system is reduced to the following items:

1. Ensure the generation of incoming transactions with an exponential distribution of arrival time intervals;

2. Describe the route of transactions:

a. place the transaction in the equipment object "control queue";

b. if further maintenance is not required - delete transact from the system;

c. move transact to the equipment object "multi-channel queuing control system";

d. to free up space in the "control queue";

e. service transact in the "multi-channel queuing control system";

f. if transact previously successfully completed a full service cycle - delete transact from the system;

g. move transact to the equipment object "multi-channel queuing system diagnostics";

$\mathrm{h}$. to make room in the "diagnostic queue";

i. service transact in the "multi-channel queuing system diagnostics";

j. place transact in the equipment object "repair queue";

k. move transaction to the object of equipment "multi-channel queuing system repair";

1. vacate the "repair queue";

m. service transact in the "multi-channel queuing system repair";

n. move transaction to the equipment object "control queue" and go to point c;

3. Ensure the maintenance of transactions in all single-channel and multi-channel queuing systems in accordance with the selected distributions.

When creating a model of a queuing system, it is necessary to take into account the fact that the probabilities of the transaction completion in the system and the exit from it at the 
time of the initial entry into the system and at the end of the cycle (after passing the service in all areas - control, diagnostics, repair, secondary control) are different.

The first probability corresponds to the entry into the system of an initially serviceable part that does not require any further maintenance, and the second to an unsuccessful service session in the system, during which the functionality of the part has not been restored; unsuccessful repair.

\section{Conclusions}

Summarizing the results of using different implementations of models, one can say about such advantages of the created software, as:

1. Visibility of the simulated queuing system.

2. Transparency and availability of the interface.

3. Ease of use.

4. No need to perform preliminary calculations when initializing parameters.

5. Output of results in an accessible and understandable form.

6. Protection against entering invalid parameters.

The disadvantages of the created software application can be attributed to the lower information content of the report (fewer characteristics of the functioning of the queuing system and statistical parameters are determined), which is explained by the specifics and requirements of the technical assignment.

At the same time, the developed program is not inferior to the model written in GPSS, in accuracy and speed.

\section{References}

1. F. N. Abu-Abed, Programmnyye produkty i sistemy, 43, 78-91 (2010)

2. F. N. Abu-Abed, V. N. Averkin, R. Y. Kordyukov, A.V. Scherbinko, Remont Vosstanovleniye Modernizatsiya, 7, 19-21 (2011)

3. T. Maertens, J. Walraevens, H. Bruneel, Queuing systems, 55:1, 27-39 (2007)

4. R. V. Dopira, A. V. Shcherbinko, D. Yu. Brezhnev, T. A. Shcherbinko, RVM, 11, 2325 (2017)

5. R. V. Dopira, D. Yu. Brezhnev, D. A. Ovrutsky, RVM, 9, 67-81 (2017)

6. R. V. Dopira, R. Y. Kordyukov, A. Y. Platonov, S. V. Sergienko, RVM, 9, 45-48 (2014)

7. T. Maertens, J. Walraevens, H. Bruneel, Queuing systems, 55:2, 90-99 (2007)

8. T. I. Aliyev, GPSS, 8, 70-75 (2013)

9. T. I. Aliyev, Theory and management system, 7, 123-138, (1987)

10. M. D. Hammer, V. N. Syzrantsev, S. L. Golofast, Simulators based on software and hardware platform for technical education (Oil and Gas University, Novosibirsk, 2011) 